Probing non-integer dimensions

This article has been downloaded from IOPscience. Please scroll down to see the full text article.

2007 J. Phys.: Condens. Matter 19065119

(http://iopscience.iop.org/0953-8984/19/6/065119)

View the table of contents for this issue, or go to the journal homepage for more

Download details:

IP Address: 128.197.40.148

The article was downloaded on 06/12/2010 at 17:40

Please note that terms and conditions apply. 


\title{
Probing non-integer dimensions
}

\author{
P L Krapivsky and S Redner
}

Center for Polymer Studies and Department of Physics, Boston University, Boston, MA 02215, USA

Received 18 September 2006

Published 22 January 2007

Online at stacks.iop.org/JPhysCM/19/065119

\begin{abstract}
We show that a two-dimensional convection-diffusion problem with a radial sink or source at the origin may be recast as a pure diffusion problem in a fictitious space in which the spatial dimension is continuously tunable with the Péclet number. This formulation allows us to probe various diffusion-controlled processes in non-integer dimensions.
\end{abstract}

\section{Introduction}

Consider a system of non-interacting passive particles that undergo a combination of diffusion and convection that is driven by a steady velocity field $\mathbf{v} \equiv \mathbf{v}(\mathbf{r})$. The particle density $c \equiv c(\mathbf{r}, t)$ obeys the equation of motion

$$
\frac{\partial c}{\partial t}+(\mathbf{v} \cdot \nabla) c=D \nabla^{2} c
$$

where $D$ is the diffusion coefficient. If the velocity field is radial, $\mathbf{v}=v(r) \hat{r}$, and the initial conditions are radially symmetric, the density satisfies

$$
\frac{\partial c}{\partial t}+v \frac{\partial c}{\partial r}=D\left(\frac{\partial^{2} c}{\partial r^{2}}+\frac{d-1}{r} \frac{\partial c}{\partial r}\right)
$$

Suppose that the velocity field is inversely proportional to the radial distance, $v=\frac{Q}{2 \pi r}$. Such a divergenceless field is natural in two dimensions and is generated by a point sink (or source) of strength $Q$ in an incompressible fluid. This flow field has the remarkable property that the convection term can be absorbed into the diffusion operator by an appropriate shift of the spatial dimension. Indeed, the density satisfies

$$
\frac{\partial c}{\partial t}=D\left(\frac{\partial^{2} c}{\partial r^{2}}+\frac{d_{\mathrm{eff}}-1}{r} \frac{\partial c}{\partial r}\right),
$$

where the effective spatial dimension is given by

$$
d_{\mathrm{eff}}=d-\frac{Q}{2 \pi D}=2-2 \lambda, \quad \lambda=\frac{Q}{4 \pi D} .
$$

Here $\lambda$ is the Péclet number; the numerical factor $(4 \pi)^{-1}$ is chosen to simplify the formulae that follow. 
We can thus interpret convection-diffusion in two dimensions with a radial velocity $v \propto r^{-1}$ as isotropic diffusion in a space with variable effective dimension. The motivation for developing this connection is that there are a variety of problems in many-body physics for which the spatial dimension is an important determining factor in the phenomenology. In diffusion-controlled reactions, for example, a large body of work has uncovered the general feature that when the spatial dimension $d$ exceeds a critical value $d_{\mathrm{c}}$ (that depends on the specifics of the reaction), the reaction kinetics has a mean-field character, a situation where any pair of reactants is equally likely to react. Conversely, when the spatial dimension $d<d_{\mathrm{c}}$, fluctuation-dominated phenomena arise, such as anomalously slow kinetics and non-trivial spatial organization of reactants (see, e.g., [1-6] for reviews). The radial flow system offers a potentially attractive way to study the full range of behaviour between the disparate meanfield and fluctuation-controlled regimes simply by tuning the flow velocity.

In this work we therefore exploit the connection between convection-diffusion in two dimensions and pure diffusion in a space with a tunable effective dimension. By this equivalence we can probe diffusion-controlled processes in an arbitrary dimension by varying the Péclet number. In the next section we emphasize some subtleties associated with this mapping. In the following sections, we study simple diffusion-controlled reactions in general dimensions by exploiting the mapping to radial flow in two dimensions. We first consider the influence of an absorbing trap on the concentration profile in section 3. In particular, we analyse the minimal separation between the particles and the trap. In section 4 we consider two simple reactive systems, namely irreversible coalescence and irreversible annihilation.

\section{Spreading of a ring}

As a warm-up exercise we first consider the spread of an initial density profile that is concentrated on a ring of radius $R$,

$$
c(r, t=0)=\frac{1}{2 \pi R} \delta(r-R) .
$$

This example illuminates some pitfalls of the mapping of convection-diffusion in two dimensions onto a pure diffusion problem and shows that the size of the ring is asymptotically relevant only for sink flows.

It is possible to find the spread of the initial ring by solving the convection-diffusion equation in two dimensions subject to the initial condition (5). The solution can be written in terms of Bessel functions in the Laplace domain or as an infinite series in the time domain. However, physical insight about the solution is more easily obtained from its asymptotic behaviour. To determine the asymptotics, we make use of the mapping (3) and express the original problem as a purely diffusive system and then use the well-known scaling solution of the latter equation to describe the spread from a point mass at the origin.

There is a subtlety in specifying the initial condition. The initial two-dimensional ring distribution (5) is normalized to unit 'mass'

$$
\mathcal{M}(t=0)=\int_{0}^{\infty} c(r, t=0) 2 \pi r \mathrm{~d} r=1 .
$$

In the effective $d$-dimensional space, however, the mass $M_{\text {eff }}$ is different,

$$
M_{\mathrm{eff}}=\int_{0}^{\infty} c(r, t=0) \Omega_{d} r^{d-1} \mathrm{~d} r=\frac{\left(\pi R^{2}\right)^{-\lambda}}{\Gamma(1-\lambda)} .
$$

Here $\Omega_{d}=2 \pi^{d / 2} / \Gamma(d / 2)$ is the surface area of a unit sphere in $d$ dimensions. Asymptotically, the concentration profile should depend on the total effective mass $M_{\text {eff }}$, while the initial size 
$R$ should become irrelevant. Thus one might anticipate that the density approaches

$$
c(r, t) \rightarrow \frac{M_{\mathrm{eff}}}{(4 \pi D t)^{d / 2}} \exp \left(-\frac{r^{2}}{4 D t}\right)
$$

in the long-time limit.

Interestingly, equation (7) is correct only for sink flows. An inconsistency in the ansatz (7) can immediately be seen by computing the total mass, $\mathcal{M}(t)=\int c(r, t) 2 \pi r \mathrm{~d} r$, in the physical two-dimensional space. Using (7) we obtain

$$
\mathcal{M}(t) \rightarrow \frac{1}{\Gamma(1-\lambda)}\left(\frac{4 D t}{R^{2}}\right)^{\lambda}
$$

in the long-time limit. Equation (8) looks reasonable for sink flows-the sink causes particles to disappear which is reflected by the decay of $\mathcal{M}(t)$. On the other hand, for source flows $(\lambda>0)$ equation (8) is invalid - the prediction that the total mass diverges as $t^{\lambda}$ is clearly nonsensical.

To resolve this puzzle, it is useful to recall the derivation of equation (7) to see why it does not apply to source flows. The fundamental solution (7) may be found by noting that the diffusion equation is invariant under the transformation $r \rightarrow a r, t \rightarrow a^{2} t$. Hence the scaling variable $\eta=r^{2} / 4 D t$ remains invariant under this scale transformation, suggesting that the fundamental solution has the form $c(r, t)=t^{-b} F(\eta)$. Then mass conservation $\int \mathrm{d}^{d} r c(r, t)=M=$ const yields $b=d / 2$. Finally, by substituting $c(r, t)=t^{-d / 2} F(\eta)$ into the diffusion equation and solving the resulting ordinary differential equation we find $F=\mathrm{e}^{-\eta}$ and thus recover equation (7).

For the radial flow problem, however, mass conservation arises in the physical twodimensional space, rather than in the effective $d$-dimensional space. Therefore, $b=d_{\text {phys }} / 2=$ 1 ; that is, the asymptotic solution should read

$$
c(r, t) \rightarrow \frac{1}{4 \pi D t} F(\eta), \quad \eta=\frac{r^{2}}{4 D t},
$$

so that the conservation law

$$
\mathcal{M}(t)=\int_{0}^{\infty} c(r, t) 2 \pi r \mathrm{~d} r=\int_{0}^{\infty} F(\eta) \mathrm{d} \eta=1
$$

indeed holds. By substituting (9) into the governing diffusion equation (3) we find

$$
\eta F^{\prime \prime}+(1-\lambda+\eta) F^{\prime}+F=0,
$$

which is solved to yield

$$
F(\eta)=\frac{\eta^{\lambda}}{\Gamma(1+\lambda)} \mathrm{e}^{-\eta}
$$

Thus for source flows, the total mass remains constant, $\mathcal{M}(t) \equiv 1$, and the density is

$$
c(r, t) \rightarrow \frac{1}{\Gamma(1+\lambda)} \frac{1}{4 \pi D t}\left(\frac{r^{2}}{4 D t}\right)^{\lambda} \exp \left(-\frac{r^{2}}{4 D t}\right) .
$$

In this case, the system 'forgets' the initial conditions.

Conversely, our hand-waving argument is correct for sink flows, as can be verified by explicit solution of the full convection-diffusion equation [6]. Therefore the total mass decays according to equation (8) and the density is given by (7), or equivalently

$$
c(r, t) \rightarrow \frac{1}{\Gamma(1-\lambda)} \frac{1}{4 \pi D t}\left(\frac{4 D t}{R^{2}}\right)^{\lambda} \exp \left(-\frac{r^{2}}{4 D t}\right) .
$$

The system 'remembers' initial conditions, as is reflected by the appearance of $R$ in the above asymptotic expressions. 


\section{Distance to the closest particle}

As an illustration of the utility of the equivalence between convection-diffusion in two dimensions and pure diffusion in general dimensions, we examine here the following question: what is the typical distance from the boundary of the absorbing trap to the closest particle? Suppose that the trap is a disc of radius $R$ centred at the origin, so that particles freely diffuse for $r>R$ and get absorbed at $r=R$. We assume that the initial density is uniform, $\left.c\right|_{t=0}=c_{0}$. We anticipate that the typical separation $r_{\text {min }}$ between the trap and the closest surviving particle exhibits the following behaviours:

- For sink flows, $r_{\min }$ is finite.

- For source flows, $r_{\text {min }}$ diverges with time.

We now confirm these expectations and establish the precise asymptotic form of the minimal distance $r_{\min }$ by employing a simple quasi-static approach [7, 8]. The minimal distance can also be found by more formal methods $[9,10]$.

For sink flows, the concentration approaches a time-independent profile $c_{\infty}(r)$ that is found by solving the Laplace equation subject to the absorbing boundary condition, $\left.c_{\infty}\right|_{r=R}=0$. This solution is

$$
c_{\infty}(r)=c_{0}\left[1-\left(\frac{R}{r}\right)^{d-2}\right] .
$$

We now estimate $r_{\text {min }}$ from the extreme-value criterion [7]

$$
\int_{R}^{r_{\min }} c(r, t) 2 \pi r \mathrm{~d} r=1
$$

which for the density profile (13) becomes

$$
1=\int_{R}^{r_{\min }} c_{0}\left[1-\left(\frac{R}{r}\right)^{d-2}\right] 2 \pi r \mathrm{~d} r .
$$

Computing the integral we find that the dimensionless minimal distance $u_{\min }=r_{\min } / R$ obeys

$$
\left(\pi c_{0} R^{2}\right)^{-1}=u_{\min }^{2}-1-\frac{u_{\min }^{2+2 \lambda}-1}{1+\lambda} .
$$

For source flows, the minimal distance grows with time because particles are driven away from the origin. In using the connection to pure diffusion in a space of dimension $d_{\text {eff }}$ we note that $d_{\text {eff }}<2$, and therefore we can set the radius of the trap to zero because a diffusing particle is recurrent and will hit a point trap with certainty if $d_{\text {eff }}<2$. This equivalent problem has the advantage of being tractable analytically by elementary means. Indeed, we look for a scaling solution of the form

$$
c(r, t)=c_{0} F(\eta), \quad \eta=\frac{r^{2}}{4 D t},
$$

that also satisfies

$$
\left.c\right|_{r=0}=0,\left.\quad c\right|_{t=0}=c_{0} .
$$

By substituting the scaling ansatz (15) into the governing diffusion equation (3) we find

$$
\eta F^{\prime \prime}+(1-\lambda+\eta) F^{\prime}=0,
$$

whose solution is

$$
F(\eta)=\int_{0}^{\eta} \mathrm{d} \xi \frac{\xi^{\lambda-1} \mathrm{e}^{-\xi}}{\Gamma(\lambda)}
$$


Using equation (14) together with (18), the minimal value $\eta_{\min }=r_{\min }^{2} / 4 D t$ of the scaling variable obeys

$$
\frac{\Gamma(\lambda)}{4 \pi D t c_{0}}=\int_{0}^{\eta_{\min }} \mathrm{d} \xi \xi^{\lambda-1}\left(\eta_{\min }-\xi\right) \mathrm{e}^{-\xi}
$$

In the long-time limit $\eta_{\min } \rightarrow 0$ and hence the above integral simplifies to $\frac{\Gamma(\lambda) \Gamma(2)}{\Gamma(\lambda+2)} \eta_{\min }^{\lambda+1}$. Thus we arrive at

$$
r_{\min } \rightarrow\left[\frac{\Gamma(\lambda+2)}{\pi c_{0}}(4 D t)^{\lambda}\right]^{1 /(2 \lambda+2)}
$$

Let us now illustrate our results by considering some specific examples. For sink flow with $\lambda=-1 / 2$, the effective dimension $d_{\mathrm{eff}}=3$ and

$$
r_{\min }=R+\left(\pi c_{0}\right)^{-1 / 2} \text {. }
$$

For source flow with $\lambda=1 / 2, d_{\mathrm{eff}}=1$ and

$$
r_{\min } \rightarrow\left(\frac{9}{4 \pi} \frac{D t}{c_{0}^{2}}\right)^{1 / 6}
$$

For source flow with $\lambda=1, d_{\mathrm{eff}}=0$ and

$$
r_{\min } \rightarrow\left(\frac{8}{\pi} \frac{D t}{c_{0}}\right)^{1 / 4}
$$

Note that for true one-dimensional diffusion, the distance from the trap to the closest surviving particle also scales as $\left(D t / c_{0}^{2}\right)^{1 / 4}[7,9,10]$. Finally for very strong source flows with $\lambda \gg 1$, the effective dimension is $d \ll 0$ and

$$
r_{\min } \rightarrow \sqrt{4 D t \frac{\lambda}{e}}=\sqrt{\frac{Q t}{\pi e}}
$$

In this case, the particles are driven away from the trap in an essentially deterministic manner and the above scaling dependence of $r_{\text {min }}$ follows directly from $\dot{r}=v \sim Q / r$.

The case of pure diffusion in two dimensions is most subtle: the density profile remains time-dependent, but we cannot seek a solution in a scaling form because the size of the trap cannot be ignored in two dimensions [6]. The divergence of the minimal distance with time is also particularly delicate in this case, namely [7, 10],

$$
r_{\min } \sim \sqrt{\frac{\ln \left(D t / R^{2}\right)}{2 \pi c_{0} \ln \left[\ln \left(D t / R^{2}\right)\right]}} .
$$

\section{Interacting particle systems with a localized source}

Consider now the influence of radial flow on an interacting particle system. Particularly simple models of this type are irreversible annihilation or irreversible coalescence [11-14]. Suppose that the system is initially empty and that a localized particle source of strength $J$ at the origin is turned on at time $t=0$. Particles are emitted at the origin and are advected by the radial flow field. The particles also diffuse and react upon colliding with each other (we set the particle radii equal to one). These processes may be described by the rate equation

$$
\frac{\partial c}{\partial t}=D\left(\frac{\partial^{2} c}{\partial r^{2}}+\frac{d_{\mathrm{eff}}-1}{r} \frac{\partial c}{\partial r}\right)-\frac{D c^{2}}{\ln (1 / c)}+J \delta(\mathbf{r}) .
$$

Here we employ a phenomenologically motivated form for the reaction term that reflects the true two-dimensional spatial nature of the problem. In the homogeneous source-free case 
equation (21) reduces to $\dot{c}=-D c^{2} / \ln (1 / c)$, which leads to $c(t) \sim(D t)^{-1} \ln (D t)$ asymptotic behaviour that was rigorously proved to be correct [15].

We now employ the same line of reasoning as that given in $[11,14]$ to determine the particle density. When the effective dimension is sufficiently large, $d>4$, particles do not 'see' each other; i.e. they interact sufficiently weakly that far from the source a non-interacting density profile, $c(r) \sim r^{-(d-2)}$, should arise. For $d<4$, the reaction and diffusion terms balance each other, leading to $c(r) \sim r^{-2} \ln r$. The borderline case of $d=4$ needs to be analysed separately, and after some algebra (see equations (30) and (31) below) it is possible to find a nested logarithmic correction to the basic $r^{-2}$ behaviour: $c(r) \sim r^{-2}[\ln (\ln r)]^{-1}$.

Having determined the density profile, we can now probe the temporal behaviour of the total number of particles. Using the fact that the flow field decays as $1 / r$ and that $\dot{r}=v$, the maximum distance travelled by particles grows as $\sqrt{t}$. Thus the total number of particle in the system scales as $N(t) \sim \int_{0}^{\sqrt{t}} \mathrm{~d} r r c(r)$. Using this relation, we arrive at the following conclusions:

- For sink flows with $\lambda<-1$, the effective dimension obeys $d_{\text {eff }}>4$. The density profile and the total number of particles are

$$
c(r) \sim r^{-(d-2)}=r^{2 \lambda}, \quad N(t) \sim 1 .
$$

The sink is thus strong enough to keep the total number of particles finite.

- For the case of sink flow with $\lambda=-1$, the effective dimension is critical, $d_{\text {eff }}=4$. The density profile and the total number of particles are

$$
c(r) \sim r^{-2}[\ln (\ln r)]^{-1}, \quad N(t) \sim \frac{\ln t}{\ln (\ln t)} .
$$

The total number of particles therefore exhibits an unusually slow growth in this marginal regime.

- For sink flows with $-1<\lambda<0$ and also for source flows, the effective dimension obeys $d_{\text {eff }}<4$. The density profile and the total number of particles become universal

$$
c(r) \sim r^{-2} \ln r, \quad N(t) \sim(\ln t)^{2} .
$$

We conclude that for the case $\lambda \geqslant-1$ the total number of particles grows extremely slowly with time, while for $\lambda<-1$ there are just a few particles in the system.

The results for source flow are seemingly peculiar; here one might naively expect that the total density grows linearly with time because particles are driven away from each other by the flow and thus would not interact. Let us therefore re-derive some of our results without exploiting the mapping between radial flow in two dimensions to pure diffusion in a variable spatial dimension. Consider, for simplicity, the case of strong source flow, i.e. the Péclet number is large. We may then drop the diffusion terms and keep just the dominant convection term. Using this simplification, and assuming that the system is in the steady-state regime, the governing equation (21) becomes

$$
\frac{2 \lambda}{r} \frac{\mathrm{d} c}{\mathrm{~d} r}=-\frac{c^{2}}{\ln (1 / c)} .
$$

Integrating this equation we obtain

$$
\frac{\ln (1 / c)}{c} \simeq A+\frac{r^{2}}{4 \lambda},
$$

where we use the shorthand notation $A=c_{0}^{-1} \ln \left(1 / c_{0}\right)$. We estimate the density $c_{0}$ near the origin from the conservation law $2 \pi r c_{0} \frac{Q}{2 \pi r}=J$ to give $c_{0}=J / Q$ and therefore

$$
A=\frac{Q}{J} \ln \left(\frac{Q}{J}\right) .
$$


From equation (26), we finally obtain the steady-state density profile

$$
c(r) \simeq \frac{\ln \left[A+\frac{r^{2}}{4 \lambda}\right]}{A+\frac{r^{2}}{4 \lambda}} .
$$

Sufficiently far from the origin, $r \gg \sqrt{\lambda A}$, equation (27) simplifies to $c(r) \sim r^{-2} \ln r$ in agreement with our previous findings in (24).

To determine the total number of particles we note that the steady-state solution formally holds as long as $r \ll \sqrt{Q t / \pi}$. Using the fact that the leading edge of the particles advances as $r=\sqrt{Q t / \pi}$, the total number of particles then scales as

$$
N(t) \sim \int_{0}^{\sqrt{Q t / \pi}} c(r) 2 \pi r \mathrm{~d} r .
$$

From (27) we then obtain

$$
N(t) \sim 2 \pi \lambda\left\{\ln ^{2}(D t+A)-\ln ^{2} A\right\} .
$$

In the long-time limit, equation (28) qualitatively agrees with our previous result, $N(t) \sim$ $(\ln t)^{2}$. Note, however, that logarithmic behaviour arises only after a short-time linear regime. For $D t \ll A$, we get $N(t) \sim 4 \pi \lambda \frac{\ln A}{A} D t$, which simplifies to $N(t) \sim J t$. Thus indeed the total density initially grows linearly in time in accordance with intuition. The crossover between these two regimes occurs at $t_{\mathrm{c}}=A / D \sim(\lambda / J) \ln (Q / J)$.

The previous analysis equally applies to annihilation and coalescence. More generally, we may also consider irreversible mass-conserving aggregation where the reaction of a cluster of mass $i$ with a cluster of mass $j$ leads to a cluster of mass $k=i+j$. For aggregation, the fundamental quantities are the densities of various particle species. Let us set the mass of particles that are emitted at the origin to unity. We denote the density of these monomer particles as $c_{1}$. Generally let $c_{k}$ be the density of particles composed of $k$ monomers. We shall seek only the stationary densities $c_{k}(r)$. These densities satisfy a modified Smoluchowski equation

$$
\left(\frac{\mathrm{d}^{2}}{\mathrm{~d} r^{2}}+\frac{d_{\mathrm{eff}}-1}{r} \frac{\mathrm{d}}{\mathrm{d} r}\right) c_{k}+\frac{1}{\ln (1 / c)}\left(\sum_{i+j=k} c_{i} c_{j}-2 c_{k} c\right)=-\frac{J}{D} \delta_{k, 1} \delta(\mathbf{r}) .
$$

Here we again employed a phenomenologically motivated form for the reaction term (see, e.g., [16]) and we additionally assumed that the coalescence rate is independent of the masses of the two reactants. (The latter assumption was originally made by Smoluchowski; it greatly simplifies the analysis of the infinite system of rate equations [17]. Furthermore, for diffusioncontrolled reactions in two dimensions, the reaction rate depends on the radii of the reactants in a weak logarithmic fashion $[1,6]$.)

The asymptotic behaviour is especially interesting in the marginal case of $\lambda=-1$ when the effective dimension is critical, $d_{\text {eff }}=4$. Then the total density satisfies

$$
\frac{\mathrm{d}^{2} c}{\mathrm{~d} r^{2}}+\frac{3}{r} \frac{\mathrm{d} c}{\mathrm{~d} r}=\frac{c^{2}}{\ln (1 / c)}
$$

whose leading asymptotic behaviour is

$$
c=\frac{4}{r^{2}} \frac{1}{\ln (\ln r)} \text {. }
$$

The density of monomers then satisfies

$$
\frac{\mathrm{d}^{2} c_{1}}{\mathrm{~d} r^{2}}+\frac{3}{r} \frac{\mathrm{d} c_{1}}{\mathrm{~d} r}=\frac{2 c}{\ln (1 / c)} c_{1}=\frac{4}{r^{2}(\ln r) \ln (\ln r)} c_{1}
$$


whose solution is

$$
c_{1} \sim \frac{1}{r^{2}} \frac{1}{[\ln (\ln r)]^{2}} .
$$

Solving for the first few $k$-mer densities one by one, we observe that they all have the form

$$
c_{k}=\frac{1}{r^{2}} F_{k}(\rho), \quad \rho=\frac{1}{4} \ln (\ln r) .
$$

This approach also gives $F_{k} \sim \rho^{-2}$ for small $k$, but it is better to derive this result in general rather than guessing this behaviour based on the small- $k$ behaviour only. Thus substituting the ansatz (33) into (29) and keeping only the dominant terms we obtain

$$
\frac{\mathrm{d} F_{k}}{\mathrm{~d} \rho}=\sum_{i+j=k} F_{i} F_{j}-\frac{2}{\rho} F_{k},
$$

which is a long-time limit of a system of equations originally solved by Smoluchowski (see [17]), with $\rho$ playing the role of time. The solution admits the scaling form $F_{k}=\rho^{-2} \mathrm{e}^{-k / \rho}$, and therefore

$$
c_{k}=\frac{16}{r^{2}} \frac{1}{[\ln (\ln r)]^{2}} \exp \left\{-\frac{4 k}{\ln (\ln r)}\right\} .
$$

Using this result we can verify that the total particle density $c(r)=\sum_{k \geqslant 1} c_{k}(r)$ is consistent with our previous prediction (31); we can also compute the mass density $m(r)=$ $\sum_{k \geqslant 1} k c_{k}(r)=r^{-2}$ and estimate the total mass to be

$$
M(t)=\int_{0}^{\sqrt{t}} m(r) 2 \pi r \mathrm{~d} r=\pi \ln t .
$$

When $\lambda>-1$, that is, the effective dimension is smaller than critical, $d_{\mathrm{eff}}<4$, the particles do not undergo a sufficient number of collisions to develop a scaling form like (34). Nevertheless, at least the monomer density always exhibits interesting dynamical behaviour. Indeed, using (29) and $c=8(1+\lambda) r^{-2} \ln r$, which is the precise form of (24) that includes the correct amplitude, we find that the monomer density satisfies

$$
\frac{\mathrm{d}^{2} c_{1}}{\mathrm{~d} r^{2}}+\frac{1-2 \lambda}{r} \frac{\mathrm{d} c_{1}}{\mathrm{~d} r}=\frac{8(1+\lambda)}{r^{2}} c_{1}
$$

Solving (36) gives

$$
c_{1} \sim r^{-n}, \quad n=-\lambda+\sqrt{\lambda^{2}+8(1+\lambda)}
$$

so that the monomer density has a pure algebraic tail with a $\lambda$-dependent exponent.

\section{Concluding remarks}

Allowing the spatial dimension $d$ to be a free parameter and then developing theoretical approaches based on this parameter (an expansion about a critical dimension, dimensional regularization, etc) has proven extremely fruitful in field theory and statistical physics [18, 19]. Thus in many treatments of critical phenomena, non-integer dimensions arise naturally, but this construction is generally used as an intermediate step toward the ultimate goal of obtaining information in physically relevant spatial dimensions, such as $d=1,2,3$. However, certain complex problems can be mapped onto simpler ones that are defined in spaces of a noninteger spatial dimension. We demonstrated that such a connection arises for a class of twodimensional convection-diffusion problems in which the flow field is radial and proportional to $1 / r$. It would be exciting to realize these convection-diffusion flows experimentally and hence probe dynamical processes in spaces whose dimension is not necessarily an integer. 


\section{Acknowledgments}

We acknowledge financial support from NSF grant CHE0532969 (PLK) and NSF grant DMR0535503 (SR).

\section{References}

[1] Ovchinnikov A A, Timashev S F and Belyi A A 1989 Kinetics of Diffusion Controlled Chemical Processes (New York: Nova Science)

[2] Redner S and Leyvraz F 1993 Fractals and Disordered Systems vol II, ed A Bunde and S Havlin (Berlin: Springer)

[3] Oshanin G, Moreau M and Burlatsky S 1994 Adv. Colloid Interface Sci. 491

[4] Privman V (ed) 1997 Non-equilibrium Statistical Mechanics in One Dimension (New York: Cambridge University Press)

[5] ben-Avraham D and Havlin S 2000 Diffusion and Reactions in Fractals and Disordered Systems (New York: Cambridge University Press)

[6] Redner S 2001 A Guide to First-Passage Processes (New York: Cambridge University Press)

[7] Redner S and ben-Avraham D 1990 J. Phys. A: Math. Gen. 23 L1169

[8] Krapivsky P L 1993 Phys. Rev. E 471199

[9] Weiss G H, Havlin S and Kopelman R 1989 Phys. Rev. A 39466

[10] Havlin S, Larralde H, Kopelman R and Weiss G H 1990 Physica A 169337

[11] Cheng Z, Redner S and Leyvraz F 1989 Phys. Rev. Lett. 622321

[12] Hinrichsen H, Rittenberg V and Simon H 1997 J. Stat. Phys. 861203

[13] Krapivsky P L 1993 Physica A 198157

[14] Krapivsky P L 1994 Phys. Rev. E 493233

[15] Bramson M and Griffeath D 1980 Z. Wahrsch. Verw. Gebiete 53183

[16] Meakin P 1990 Physica A 1651

[17] Chandrasekhar S 1943 Rev. Mod. Phys. 151

[18] Wilson K G and Fisher M E 1972 Phys. Rev. Lett. 28240

Wilson K G 1972 Phys. Rev. Lett. 28548

[19] Itzykson C and Drouffe J-M 1989 Statistical Field Theory (Cambridge: Cambridge University Press) 